\title{
2 Wohnpolitik und Städtebau in der Sowjetunion
}

\section{Stadtplanung und Wohnpolitik von der Oktoberrevolution bis Khrushchev - ein chronologischer Überblick}

Vor der Beschäftigung mit dem eigentlichen Beispiel ist es sinnvoll, auf städtebauliche Prozesse zu schauen, die sich in der ganzen Sowjetunion abgespielt haben, um den nötigen Kontext nicht aus den Augen zu verlieren.

Wohnungsmangel war zweifellos eines der dringlichsten sozialen Probleme in der Sowjetunion, und es bestand vom ersten bis zum letzten Tag ihrer Existenz - mit unterschiedlicher Intensität und mit unterschiedlichen Lösungsstrategien. Die Themen Städtebau und Wohnungsfrage hängen unmittelbar zusammen, wurden jedoch in der Sowjetunion nicht immer zusammen gedacht und erfuhren in jedem Jahrzehnt eine andere Konzeption und Priorität.

Die gesetzlichen Grundlagen für einige Besonderheiten der sowjetischen Wohn- und Städtebaupolitik entstanden bereits in den ersten Tagen und Monaten des sowjetischen Staates. Einer der ersten Erlasse der jungen Sowjetmacht war das „Dekret über den Boden“ vom 28. Oktober 1917. ${ }^{30}$ Dieses schaffte das Recht auf Eigentum an Grund und Boden ab, übertrug den gesamten Boden in den so genannten Volksbesitz und überließ die Verwaltung den lokalen Räten. 1936 wurde die Abschaffung des Bodenbesitzes auch in der Verfassung festgehalten; ${ }^{31}$ sie blieb darin bis zum Ende der UdSSR festgeschrieben. So genossen später die Stadtplaner bei großen Projekten, im Gegensatz zum Beispiel zu ihren westeuropäischen Berufskollegen, viel größere Freiheiten, da der sowjetische Staat keine Rücksicht auf Bodenbesitz nehmen musste. ${ }^{32}$

Auf der Ebene der Wohnpolitik verstaatlichte der junge sowjetische Staat in den Jahren 1917-1922 mit zahlreichen Beschlüssen etappenweise große Mengen an bis dahin privatem Wohnraum und übernahm die Kontrolle über die Wohnraumverteilung. Inmitten von Revolution und Bürgerkrieg entstand kaum neuer

30 Durch die Kalenderreform findet man in den Quellen unterschiedliche Angaben: 26. Oktober nach dem vorrevolutionären Julianischen Kalender, 8. November nach dem heute noch gültigen Gregorianischen.

31 Artikel 6 der Verfassung der Sowjetunion von 1936.

32 Smith 2010, S. 18.

Ә Open Access. (C) 2021 Marya Petrova, publiziert von De Gruyter. (c) BY-NC-ND Dieses Werk ist lizenziert unter der Creative Commons Attribution-NonCommercial-NoDerivatives 4.0 International Lizenz.

https://doi.org/10.1515/9783110669367-002 
Wohnraum, daher bestand die Wohnpolitik des jungen Revolutionsstaates primär in der Umverteilung des enteigneten Wohnbesitzes. ${ }^{33}$ Besonders anschaulich ist hier das Beispiel von Moskau, das für die neue Regierung als eine Art Experimentierfeld funktionierte. Bereits im Dezember 1917 wurde in Moskau Wohneigentum, dessen Wert bzw. jährlicher Ertrag aus der Vermietung über eine bestimmte Summe hinausging, enteignet. Zwei Erlasse des Moskauer Sowjets aus dem Herbst 1918 ,Über die Verfahrensweise der Requirierung von Wohnraum und beweglichem Eigentum“ und „Über die Erfassung und Verteilung des bewohnten und unbewohnten Raums in Moskau“ führten die bereits begonnene Umverteilung des Wohneigentums weiter. ${ }^{34}$ So wurden Personen oder Familien, deren Wohnfläche die festgelegte „sanitäre Norm“ von ca. $8 \mathrm{~m}^{2}$ pro Erwachsenen ${ }^{35}$ überstieg, „verdichtet“36 - das heißt, ihnen wurden zwangsweise Mitbewohner zugeführt oder, falls als besonders „parasitierend“ oder bourgeois betrachtet, wurden die Menschen ausquartiert und mussten Moskau verlassen. Aleksei Fedorov schreibt von mehr als 3.000 Familien, die dieses Schicksal traf. ${ }^{37}$ Die Umsiedlung in Häuser der Bourgeoisie begünstigte vor allem Arbeiter und Mitarbeiter der wachsenden Verwaltung des jungen Staates: Kommunalwohnungen wurden geschaffen.

Zweifellos bedeutete diese Umverteilung für Einige etwas bessere Wohnbedingungen als vorher; sie führte aber zu keinem Wohnraumzuwachs, im Gegenteil: Infolge dieser Maßnahmen gingen bis zu 34\% des Wohnraumbestandes an den wachsenden bürokratischen Apparat über, wodurch sich die Situation noch mehr verschärfte. ${ }^{38}$ Dazu kam die Überforderung der neuen städtischen Verwaltungen, die mit den Folgen von Revolution und Krieg sowie dem Zusammenbrechen von Versorgung und Produktion, welche die Stadt- und Wohnwirtschaft aufrecht erhalten sollte, zu kämpfen hatte, was zu katastrophalen Zuständen führte. ${ }^{39}$

Relativierend wirkt der Umstand, dass dieser Prozess das größte Ausmaß vor allem in Moskau, St. Petersburg (damals Petrograd) und einigen anderen großen Städten annahm. In der Provinz fiel der Anteil des verstaatlichten Wohnraums

33 Andrusz 1984, S. 13.

34 Fedorov 2010, S. 240, sowie 244-245.

35 Meerovich 2014, S. 98

36 Eine direkte Übersetzung des russischen Begriffs uplotnenie, der in dieser Zeit aufkam.

37 Fedorov 2010, S. 245.

38 Kozenrenko 1928, zitiert nach Andrusz 1984, S. 14. Fedorov schreibt z.B. von 25\% Verlust durch Krieg, Revolution und Verfall und 10\% durch Umnutzung.

39 Fedorov 2010, S. 246-251. 
viel geringer aus. ${ }^{40}$ Fedorov schreibt auch, dass der NKVD, der zu diesem Zeitpunkt für die Wohnpolitik zuständig war, abgeschreckt von den Ergebnissen der Moskauer „flächendeckenden Verstaatlichung“ von einer konsequenten Abschaffung des Wohnbesitzes in den Städten, so wie sie das Dekret vom August 1918 eigentlich vorsah, Abstand nahm. ${ }^{41}$ Dies erklärt die Entstehung des praktischen Kompromisses der Revolutionsjahre, der sich später in der juristischen Formel des „individuellen Eigentums“ etablierte. ${ }^{42}$

Die „neue Wohnpolitik“ in den Zeiten der Neuen Ökonomischen Politik von 1921 bis 1928 erlaubte eine teilweise Rückübernahme der verstaatlichten Häuser durch ihre Vorbesitzer sowie die Vermietung von Wohnraum. Zudem wurden selbstverwaltete Wohngemeinschaften zugelassen, womit teilweise eine wenn auch stark beschränkte Wiederherstellung von privatem Eigentum möglich wurde. ${ }^{43}$ Die Besitzer wurden verpflichtet, für die Erhaltung ihres Wohnraums zu sorgen. Im Jahre 1924 wurde eine Form von Wohnkooperativen ins Leben gerufen, die die Anmietung oder den Bau von Wohnungen zum Ziel hatten; diese wurden aber 1937 wieder abgeschafft und ihr Wohnraumbestand ging in den Besitz der lokalen Verwaltung über. ${ }^{44}$ Damit nahm sich der Staat die Rolle des einzigen Organs, das über die Wohnraumverteilung bestimmte, zurück.

Des Weiteren wurde im Laufe der 1920er-Jahre das sowjetische Mietrecht herausgearbeitet: Nach einer kurzen Phase der kompletten Mietfreiheit (1921-1922) wurde ein Tarifsystem eingeführt, das die Höhe der Miete von der Qualität des Wohnraums, vom Einkommen und von der sozialen Klasse des Mieters abhängig machte und dabei insbesondere die Arbeiter begünstigte. Die Berechnung der konkreten Satzhöhe pro Quadratmeter wurde lokalen Sowjets überlassen; der Höchstsatz wurde zentral festgelegt und blieb bei 13,2 Kopeken pro Quadratmeter, womit allerdings die laufenden Kosten nicht gedeckt werden konnten. Das Gesetz wurde zwar im Laufe der Jahrzehnte angepasst, in seinem Wesen und in der Methodik sowie der Größenordnung der Tarife blieb es jedoch bis ans Ende der Sowjetunion gültig, sodass eine Familie beispielsweise im Jahr 1961 lediglich ca. 3-4 Prozent ihres monatlichen Einkommens für Wohnen ausgeben musste. ${ }^{45}$

40 Andrusz 1984, S. 14.

41 Fedorov 2010, S. 252.

42 Mehr dazu im nächsten Unterkapitel.

43 Vereshchak 2011, S. 209. Der Staat gab meist sehr heruntergekommene Wohnungen und Häuser „zurück“ an die Besitzer mit der Auflage, sie zu renovieren. Außerdem blieb das Land, also der Boden, ab der Nationalisierung im Jahr $1917 \mathrm{im}$ Besitz des Staates.

44 Erlass des Exekutivkomitees SNK vom 17.10.1937.

45 Andrusz 1984, S. 28 und S. 299 Fn. 5. 
Die niedrigen, ja fast symbolischen Mieten waren ein wichtiges Propagandaargument für die sowjetische Führung im Vergleich mit den westlichen kapitalistischen Staaten, mit dem erklärten Ziel, Mieten in der kommunistischen Zukunft komplett abzuschaffen. ${ }^{46}$ Das Festhalten an den niedrigen Mieten sorgte dafür, dass der staatliche Wohnungssektor ein hoch subventionierter und chronisch unterfinanzierter Bereich blieb, was sich in seiner Qualität niederschlug.

Thomas Bohn erinnert daran, dass es keine marxistischen Vorgaben für eine sozialistische Stadt gab, sodass die Konzeption der sowjetischen Städte den Stadtplanern und Ideologen überlassen war. ${ }^{47}$ Dementsprechend wandelten sich auch Verständnis und Idealbild einer Stadt im Laufe der Jahrzehnte. Die 1920erJahre waren von unterschiedlichen utopischen Vorstellungen und Diskussionen über die Gestaltung der Städte und des neuen sozialistischen Wohnens geprägt. Als Beispiel für eine frühe utopische Sichtweise können die „Kommunenhäuser“ genannt werden - diese versuchten mit Hilfe architektonischer Lösungen auf Basis eines kollektivistischen Lebensmodells mit dem konventionellen Familienbild zu brechen, für die Emanzipation der Frau zu sorgen und einen neuen, sowjetischen Typ Menschen zu schaffen und zu erziehen. ${ }^{48}$ Eine weitere Diskussion fand zwischen den so genannten „Urbanisten“ und „Desurbanisten“ statt. Die Letzteren begründeten in ihren theoretischen Schriften die Notwendigkeit der Begrenzung des städtischen Wachstums und forderten die Auflösung der Städte und eine Dispersion der Industrie im Land in dem Bestreben, mit dem bourgeoisen, individualistischen Leben $\mathrm{zu}$ brechen, das die Städte provozierten. ${ }^{49}$ Die meisten dieser Ideen gelangten jedoch nicht über das Stadium des Entwurfs hinaus und wurden Anfang der 30er-Jahre von einer neuen Tagesordnung überholt.

Zur Zeit der stalinistischen Herrschaft hatte die Industrialisierung oberste Priorität, weswegen nur ungenügend Investitionen für den Wohnungsbau vorgenommen wurden. Gleichzeitig fand auf der konzeptionellen Ebene eine Abkehr von utopischen Gesellschaftsbildern statt, hin zu einer pragmatischen Sichtweise, welche sich in Versuchen äußerte, das Wachstum der Städte zu begrenzen und die Infrastruktur zu verbessern. ${ }^{50}$ Dabei waren es vor allem die Eliten und für besondere Verdienste belohnte Arbeiter, die in den wenigen dieser Wohnungen

46 Programm der KPdSU von 1961, Abschnitt II.

47 Bohn 2008, S. 4.

48 Ausführlicher über die sowjetische Architekturavantgarde siehe A. Kopp 1970.

49 Bohn 2008, S. 33.

50 Bohn 2008, S. 34. 
untergebracht wurden. So konnte Wohnraum leicht als Mittel zur Kontrolle benutzt werden - er wurde zur Belohnung erteilt und als Bestrafung entzogen. ${ }^{51}$ Als Orientierung, insbesondere bei Stadtneugründungen, galten für die Architekten früher herausgearbeitete Prämissen: eine Gliederung der Stadt in unterschiedliche Zonen für Wohnen, Arbeiten und Erholung - und ausreichend Grünanlagen und Licht. ${ }^{52}$ Als Beispiel und Leitlinie für viele große Städte wurde lange der Moskauer Generalplan von 1935 und damit seine kreis-radiale Anordnung der Straßen betrachtet. ${ }^{53}$ Gebaut wurden vor allem repräsentative Straßenzüge und Plätze im monumentalen neoklassizistischen Stil, passend für Paraden und Großdemonstrationen, während die Schaffung einer ausreichenden Menge an Wohnraum keine Priorität hatte.

Durch die Zerstörungen im Zweiten Weltkrieg verlor die Sowjetunion ein Sechstel des gesamten städtischen Wohnraumbestandes; hinzu kam noch einmal so viel an beschädigten Wohnungen. ${ }^{54}$ Damit wurde die bereits davor herrschende Wohnungsnot enorm, ja katastrophal. In Städten, die weiter von den gefährdeten Grenzen entfernt lagen, z.B. am Ural oder in den Republiken Zentralasiens, war die Situation ebenfalls schwer, da diese Regionen Tausende Evakuierte aus den Kriegsgebieten aufgenommen hatten. Die Baumaßnahmen, die daraufhin getroffen wurden, überstiegen zwar die Vorkriegszahlen, konnten aber den Bedarf nur sehr rudimentär decken, was die Menschen an den Rand der Verzweiflung trieb. Davon zeugen zahlreiche Beschwerden der Sowjetbürger an alle nur möglichen Instanzen. ${ }^{55}$ Die Gründe dafür lagen sowohl in der unzureichenden und schlecht koordinierten Finanzierung - selbst nach dem Krieg wurde dem Wohnungsbau nämlich durch Stalin nicht oberste Priorität zugewiesen ${ }^{56}$ - als auch in der zerstörten Bauindustrie und im Fehlen von Arbeitskräften. Dennoch,

51 Meerovich 2014, S. 97. Smith schreibt aber dazu, dass die Wohnraumknappheit weniger ein bewusstes Mittel der Kontrolle und Disziplinierung war, sondern dass sie als Nebenprodukt der gegebenen Ressourcenknappheit bzw. einer anderen Prioritätensetzung zu verstehen ist (Smith 2010, S. 186, Fn.14).

52 Viele dieser Vorstellungen waren keine genuin sowjetischen, vielmehr haben sie ihren Ursprung in den Ideen zur Modernisierung des Städtebaus sowie des sozialen Wohnungsbaus in Westeuropa, die in den 1920er-30er-Jahren in Organisationen wie CIAM (Congrès Internationaux d'Architecture Moderne), in die auch sowjetische Architekten involviert waren, entwickelt wurden (Mumford 2009).

53 Bohn 2008, S. 303.

54 Andrusz 1984, S. 19.

55 Smith 2010, S. 178. Die Wiederaufbaumaßnahmen nach dem Krieg beschreibt Smith als „simultaneously unprecedented and dreadfully inadequate“.

56 Smith 2010, S. 32-33. 
so Smith, wurden gerade in diesem Nachkriegschaos Ideen und Strategien entwickelt und damit die Voraussetzungen geschaffen, die es Khrushchev später ermöglichen sollten, sein Massenwohnungsbauprogramm umzusetzen. Smith beschreibt dies als ,a latent proto-programme“, in dem zahlreiche große und kleine Prozesse und Initiativen sowohl im Zentrum als auch lokal, nicht zentralisiert und meist unbewusst, trotz der fehlenden Priorisierung dazu beitrugen, dass nach und nach die industrielle Basis ausgebaut, die Organisationsstruktur der Wohnungswirtschaft reformiert und Finanzierungsmodelle sowie die Anreize für Arbeitskräfte verbessert wurden. ${ }^{57}$

Eines der erfolgreichsten Programme des Wiederaufbaus nach dem Krieg hat unmittelbare Bedeutung für die vorliegende Arbeit: es handelt sich um die Unterstützung und Förderung von individuellem Hausbau, welche sich in zwei Gesetzen von 1944 und von 1948 manifestierte. Das erste ordnete ein Programm zur Wiederherstellung des Wohnraumbestandes in befreiten Gebieten an, und das zweite behandelte die weitere Reglementierung von Kauf und Bau von individuellen Häusern..$^{58}$ In der Folge machte der individuelle Bereich in den Jahren 19461949 im Schnitt 30 Prozent des gesamten Neubaus von Wohnraum aus, und in einigen Städten, z.B. in der Ukraine, erreichte der Anteil sogar bis zu 70 Prozent. ${ }^{59}$ Auf Bedeutung und Umfang des individuellen Bauens werde ich im übernächsten Abschnitt ausführlicher eingehen.

In der Amtszeit von Khrushchev, der nach Stalins Tod im Jahr 1953 Generalsekretär der KPdSU wurde, änderte sich die Prioritätensetzung. Zuerst wurde im Jahr 1955 eine Revision aller Projekte in Hinblick auf „überflüssige“ Gestaltungsoder Dekorelemente gefordert mit dem Ziel, die Baukosten möglichst zu reduzieren. ${ }^{60}$ Im selben Jahr wurde eine Dezentralisierung der Stadtplanung durchgesetzt, indem die Verantwortung dafür an die Unionsrepubliken abgegeben wurde. Gleichzeitig wurde die Anwendung industrieller Methoden im Bauwesen vorgeschrieben. ${ }^{61}$

Das Jahr 1957 markiert den Start des unionsweiten Wohnungsbauprogramms und den Startschuss für das industrielle Bauen. Das Versprechen, jede Familie innerhalb von zwölf Jahren mit einer individuellen Wohnung auszustatten, wird zu einem der wichtigsten politischen Ziele des nächsten Jahrzehnts. ${ }^{62}$ In Bezug

57 Smith 2010, S. 52-58 und 179.

58 Erlass des Präsidiums des Obersten Rates der UdSSR vom 26.08.1948.

59 Andrusz 1984, S. 99.

60 Beschluss des ZK der KPdSU vom 04.11.1955.

61 Bohn 2008, S. 303.

62 Erlass des Zentralkomitees und Ministerrates der UdSSR vom 31.07.1957 „Über die Entwicklung des Wohnungsbaus in der UdSSR“. 
auf den individuellen Hausbau war die Politik des sowjetischen Staates, wie diese Arbeit zeigen wird, von Pragmatismus und Kosten-Nutzen-Überlegungen geprägt, indem für die Lösung des Wohnraummangels auch individuell vorhandene Mittel der Bevölkerung herangezogen wurden. Bereits 1962 wird die Regelung zum individuellen Bauen jedoch verschärft: Die Zuteilung von Parzellen und Krediten für das individuelle Bauen wird in den Hauptstädten der Unionsrepubliken verboten; die Ministerräte der Republiken behalten aber das Recht, über die Handhabung des individuellen Bauwesens selbst zu entscheiden. An die Stelle des individuellen Bauens soll nun die Förderung von Baukooperativen treten. ${ }^{63}$ Dabei handelte es sich um den Bau von Wohnblöcken, in denen die Bürger eigene Wohnungen haben sollten.

Zeitgleich setzte sich in der stadtplanerischen und städtebaulichen Praxis die Idee des mikroraion (Mikrobezirks) durch, einer strukturierenden Planungs- und Baueinheit für Wohngebiete in den Städten. Für eine bestimmte Zahl von Bewohnern festgelegt, vereinigten diese Einheiten vier- bis fünfgeschossige Plattenoder Backsteinbauten und Objekte der öffentlichen Infrastruktur wie Schulen, Kindergärten und Einkaufsmöglichkeiten, deren Anordnung und Kapazitäten festgelegten Standards und Berechnungen folgte - eine Planungsmethode, die das Antlitz zahlreicher Ostblockstädte prägen sollte. Diesen Massenwohnungsbau und die Organisation der Zuteilung an die Bevölkerung beschreibt Smith als eine der umfangreichsten und aufschlussreichsten Wohlfahrtsmaßnahmen der SU und als eine der größten sozialen Reformen in der europäischen Geschichte. ${ }^{64}$ Während die vier- bis fünfstöckigen Etagenbauten sprichwörtlich für das damalige Wohnungsbauprogramm stehen, ist der gleichzeitige individuelle Wohnungsbau viel weniger bekannt. Von Ausmaß und Bedeutung des Letzteren sowie von den rechtlichen Aspekten wird in den folgenden Abschnitten die Rede sein.

\section{Das Haus als individuelles Eigentum in der Sowjetunion}

Es ist bereits deutlich geworden, dass das Wohnungsbauprogramm, das in der Regel mit Khrushchev assoziiert wird, schon unter Stalin nach dem Zweiten Weltkrieg ansetzte und dass der individuelle Hausbau bei der Entschärfung des Wohnraummangels eine große Rolle spielte - stellenweise gefördert und unter-

63 Erlass des ZK der KPdSU vom 01.06.1962. „Über den individuellen und kooperativen Wohnungsbau“.

64 Smith 2010, S. 151. 
stützt durch den Staat. Diese Erkenntnis mag zuerst als ein Widerspruch erscheinen: zu stark ist das Image der Sowjetunion als kollektivistischer Staat. Der Umstand, dass der Boden tatsächlich komplett verstaatlicht war, hat zusammen mit den Enteignungen der 1920er- und 30er-Jahre zu dieser Sichtweise beigetragen. Angesichts des Umfangs und der Bedeutung, die der individuelle Hausbau in der UdSSR spielte, stellt sich natürlich die Frage nach dem rechtlichen Status dieser Eigentumsform. Auf diese Aspekte geht dieses Kapitel ein und es wird sich zeigen, wie die Frage des privaten Wohneigentums im sowjetischen Recht gelöst wurde und wie sich die Praxis gestaltete.

Insgesamt existierten im sowjetischen System vier Formen des Wohneigentums: sozialistisches, also Eigentum der Sowjets (Räte); betriebliches oder behördliches Eigentum (vedomstvennaya sobstvennost' ${ }^{6}{ }^{65}$; kooperatives Eigentum; und als letztes und für diese Arbeit relevantes: persönliches Eigentum (lichnaya sobstvennost'), also Eigentum der Bürger. Bei den privat gebauten Häusern handelte es sich genau um diese letztere Eigentumsform.

Es ist kein Zufall, dass dieser Eigentumstyp nicht als „privates Eigentum“ chastnaya sobstvennost', sondern als „persönliches Eigentum“, also lichnaya sobstvennost', bezeichnet wurde. Diese Sonderform wurde im sowjetischen Recht nach der Abschaffung des Privateigentums unmittelbar nach der Oktoberrevolution herausgearbeitet, was aber nicht das komplette Verschwinden aller Formen privaten Eigentums bedeutete. ${ }^{66}$ Die Bestimmungen zum individuellen Wohneigentum setzten sich aus diversen Dekreten, Gesetzen und letztlich Einträgen in der Verfassung der UdSSR von 1936 zusammen. ${ }^{67}$ Demnach durfte ein Bürger ein Haus als persönliches Eigentum besitzen und hatte das Recht, es zu verkaufen, $\mathrm{zu}$ verschenken, $\mathrm{zu}$ vererben und sogar $\mathrm{zu}$ vermieten, das alles allerdings mit strengen Auflagen: Je nach Gesetzeslage durfte das Haus eine bestimmte Anzahl von Zimmern oder Quadratmetern nicht überschreiten und nicht zur Generierung von so genannten „Einkünften, die nicht durch Arbeit erzielt werden“ (netrudovye dokhody) dienen, die Miete durfte also nicht die staatlich festgelegten Höchstsätze überschreiten. ${ }^{68}$

Diesen Einschränkungen liegt der Unterschied zugrunde, den die sowjetischen Rechtswissenschaftler zwischen privatem und persönlichem Eigentum machten: Während das eine als kapitalistische Eigentumsform gedacht wurde, die zum Gewinn von Kapital und zur Ausbeutung von Menschen diente, war das

\footnotetext{
65 Als vedomstvo konnten Behörden, Organisationen oder industrielle Betriebe gelten.

66 Smith 2010, S. 18.

67 Smith 2008, S. 284, fn. 6.

68 Smith 2008, S. 285 und Andrusz 1984. S. 102.
} 
individuelle Eigentum lediglich zum persönlichen Gebrauch gedacht, wie Kleidung oder Möbel, ein Produkt eigener Arbeit, also „ehrliches“, „verdientes“ Gut. Das Wohneigentum wurde so auf reines „Nutzeigentum“ reduziert und dadurch in Einklang mit der sozialistischen Moral gebracht. ${ }^{69}$ Was nicht vergessen werden darf: Der Boden blieb dabei stets Eigentum des Staates und konnte jederzeit bei Bedarf, z.B. für staatliche Bauvorhaben, wieder entzogen werden. Im Regelfall wurde er aber bei der Vergabe von Parzellen zu einer „unbefristeten Nutzung“ (bessrochnoe pol'zovanie) übergeben.

Den eigentlichen Ursprung dieser Eigentumsform und der Praktiken des Umgangs damit sieht Mark Smith in einem „praktischen revolutionären Kompromiss“70, denn wie bereits oben beschrieben hätte die neue revolutionäre Verwaltung gar nicht die Kapazitäten und Ressourcen gehabt, den gesamten Wohnraumbestand $\mathrm{zu}$ verwalten. Zudem zeigten die offensichtlich abschreckenden Beispiele von Moskau und Petrograd, was eine totale Verstaatlichung bedeuten würde. So blieben bis in die 1960er-Jahre hinein 30 Prozent des Wohnraumes in der UdSSR in privaten Händen. ${ }^{71}$

Smith betrachtet den sowjetischen Wohnungsbau - sowohl den staatlichen als auch den individuellen - aus der Perspektive der Leistungen eines Wohlfahrtsstaates, in dem Sozial- und Eigentumsrechte im Zuge des Nachkriegsaufbaus einander stärkten. Mit dem Wohnungsbauprogramm von Khrushchev kamen unterschiedliche Formen des individuellen Eigentums zustande, die einen für die UdSSR spezifischen Nexus bildeten. ${ }^{72}$ Die Besonderheit des sowjetischen Modells, das Smith beschreibt, beinhaltet nicht nur den Erhalt und Aufbau in großem Umfang von de jure individuellem Eigentum, sondern auch von staatlichem Wohnraum, der zwar offiziell vermietet wurde, de facto aber Eigenschaften des Eigentums besaß:

[...] the Soviet Union was distinctive in allocating a high level of individual ownership to residents who, by law, were tenants. In (Western) welfare states, state and private tenants held much more limited rights of possession, and no real rights of ownership at all. ${ }^{73}$

Zwar bezieht sich Smiths Argumentation auf die gesamte Sowjetunion, als Hauptbeispiel dient ihm aber die RSFSR. Jedoch unterschied sich die Gesetzgebung unter den Unionsrepubliken. Ein interessantes Beispiel hierfür liefert der

69 Smith 2008, S. 285.

70 Smith 2010, S. 143.

71 Andrusz 1984, S. $100 \mathrm{ff}$.

72 Smith 2008, S. 286.

73 Smith 2010, S. 150. 
Zivilkodex der Usbekischen Sowjetrepublik, der zuließ, dass eine Person nicht nur als Miteigentümer des ganzen Hauses eingetragen wurde, sondern als alleiniger Eigentümer eines konkreten Teils des Gebäudes gelten konnte. So war in Artikel 134 des Zivilkodex der UzSSR vorgesehen, dass die Eigentümer berechtigt waren, eine Teilung des Hauses unter Berücksichtigung der ihnen gehörenden Teile durchzuführen, sodass bei jedem von ihnen ein separater Hausteil (Wohnung oder abgetrenntes Zimmer) als individuelles Eigentum eingetragen werden konnte. $^{74}$

Mit dieser besonderen Regelung wurde wahrscheinlich dem Umstand Rechnung zu tragen versucht, dass in vielen Fällen verheiratete Söhne im Wohnverband mit den Eltern blieben, gleichwohl aber einen eigenberechtigten Hausstand haben sollten.

\section{Individueller Wohnungsbau nach dem Zweiten Weltkrieg}

Wie bereits erwähnt, führten die Zerstörungen durch den Zweiten Weltkrieg und die katastrophale Wohnungsnot danach unter anderem dazu, dass die individuelle Bautätigkeit eine explizite Förderung und Unterstützung durch den Staat bekam. Im untersuchten Zeitraum der 1950er- und 60er-Jahre blieb die Einstellung der Politik zu dieser Art Wohnungsbau jedoch nicht konstant. Für die Phase der intensiven Förderung ist die Gesetzgebung der Jahre $1944^{75}$ und $1948^{76}$ maßgebend. Der Erlass von 1944, bereits ein Jahr vor Kriegsende, schrieb unter anderem eine im Vergleich zu der Bestimmung aus dem Jahr 1939 großzügigere und breitere Grundlage für die Vergabe von Krediten vor (zehn- statt fünftausend Rubel, und dies für die Dauer von maximal sieben Jahren im Vergleich zu den bisherigen fünf, für bestimmte Berufsgruppen sogar bis zu zehn Jahren). In seinem Titel bezieht sich der Erlass direkt auf den Wiederaufbau der vom Krieg zerstörten Gebiete. Es ist anzunehmen, dass er zuerst vor allem in diesen Gegenden zur Anwendung kam. Während individueller Hausbau in den Jahren 1946-1949 im Durchschnitt 30 Prozent des gesamten neu errichteten Wohnraumbestandes ausmachte, ging der Anteil in einigen Gegenden der Ukraine (z.B. im heutigen Lugansk) bis an die 70 Prozent, und selbst in Kiew waren es 43 Prozent. ${ }^{77}$ Andere statistische Daten zeigen, dass das Kreditprogramm bereits in den ersten Jahren

74 Vedomosti Verkhovnogo Soveta UzSSR. 1963, Nr. 9, S. 30.

75 Erlass des SNK vom 29.05.1944.

76 Verordnung des Präsidiums des Obersten Sowjets der UdSSR vom 26.08.1948.

77 Andrusz 1984, S. 99. 
nach dem Krieg aktiv genutzt wurde: Wohnungsbaukredite machten den größten Anteil bei der Kommunalbank aus. ${ }^{78}$

Im Beschluss von 1948 fehlt der Bezug zum Wiederaufbau nach dem Krieg; vielmehr war sein Ziel, Gesetzgebung und vorhandene Regelungen zu vereinheitlichen und das Recht auf Hausbau und Wohnraumerwerb zu verankern. Damit durfte jeder sowjetischer Bürger ein individuelles Haus (ein- bis zweistöckig, maximal fünf Zimmer groß) bauen. Mit kleineren Korrekturen blieb dieser Beschluss in den darauf folgenden Jahrzehnten die rechtliche Grundlage für den individuellen Hausbau. Smith bezeichnet die in der Zeit zwischen 1944 und 1950 umgesetzten bzw. verabschiedeten Bauprogramme als „the first substantial government-directed housing construction policy in either Soviet or Russian history“ “ ${ }^{79}$

Die gesamtstaatlichen Investitionen in den Wohnungsbau verdoppelten sich in der fünften (1951-1955) und sechsten (1956-1960) Planperiode jeweils und erreichten damit 23,5 Prozent, also knapp ein Viertel, des staatlichen Budgets. ${ }^{80}$ Davon profitierte auch der individuelle Wohnungsbausektor, der ebenfalls durch die 1950er-Jahre hindurch ein wichtiger Bestandteil der sowjetischen Wohnpolitik blieb und mit einer pragmatischen und flexiblen Einstellung seitens der Politik betrieben wurde. $\mathrm{Zu}$ diesem Schluss kommt Mark Smith, indem er die Republikhauptstädte sowie verschiedene große und mittelgroße Städte der UdSSR vergleicht und unterschiedliche Entwicklungen im individuellen Hausbausektor im Zeitabschnitt von 1940 bis 1960 feststellt. Er beobachtet eine Bandbreite an Variationen in Bezug auf die Anzahl der gebauten individuellen Häuser, und zusammen mit anderen Daten leitet er davon eine Situation großer Flexibilität ab, in der die städtischen Verwaltungen und Betriebe je nach den zur Verfügung stehenden Möglichkeiten sowohl auf die eine als auch auf die andere Form des Wohnungsbaus zurückgreifen konnten, um der Wohnungsnot in ihren Städten zu begegnen. ${ }^{81}$

Zusätzlich verpflichtete der Staat lange Zeit verschiedene Betriebe und Einrichtungen, einen Beitrag zur Beseitigung der Wohnungsnot zu leisten. Sie wurden angeregt oder gezwungen, de facto Sozialleistungen für ihre Beschäftigten in Form von Wohnungen in eigens dafür gebauten Häusern oder in Form von Baumaterialien für individuell $\mathrm{zu}$ errichtende Häuser zur Verfügung $\mathrm{zu}$ stellen. ${ }^{82}$

78 Smith 2008, S. 287-288.

79 Smith 2010, S. 36.

80 Andrusz 1984, S. 19.

81 Smith 2010, S. 90-91.

82 Smith 2010, 38 ff. 
Gleichzeitig standen Stadtverwaltungen und Betriebe vor den gleichen organisatorischen, finanziellen und technischen Problemen wie der Rest des Wohnungsbausektors und konnten daher den Anforderungen nur bedingt nachkommen. Das Plansoll wurde dennoch stets sehr hoch angesetzt - so wurden für die Jahre 1956-1960 individuelle Häuser im Umfang von 113 Millionen Quadratmetern geplant, was 34 Prozent des gesamten neu gebauten Wohnraums ausgemacht hätte. ${ }^{83}$ Diese ambitionierten Zahlen zeigen, welche Bedeutung dem individuellen Wohnungsbau im Gesamtrahmen des unionsweiten Wohnungsbauprogramms von 1957 beigemessen wurde.

Durch die intensive Förderung erreichte der private Sektor in den Jahren 1956-1960 zwar nicht die angestrebten 34 Prozent, immerhin aber eindrucksvolle 25 Prozent der gesamten Neubauten im städtischen Raum. Das Jahr 1960 markiert den Höhepunkt, indem der Anteil der individuellen Bauten am Gesamtwohnraumbestand der Union 39,1 Prozent erreichte. ${ }^{84}$ Diese Daten entstammen dem von Gregory Andrusz zusammengestellten Überblick über die Dynamiken im Verhältnis zwischen privatem und staatlichem Wohnraum, aufgeteilt nach Republiken. Darin fällt auf, dass in der Usbekischen SSR im Jahr 1960 der private Wohnraum den höchsten Anteil innehatte, nämlich 63,7 Prozent. Taschkent hatte dabei nach Angaben des damaligen Hauptarchitekten von Taschkent, Bulatov, den größten Prozentanteil an privaten Häusern unter den großen Städten in der gesamten Sowjetunion. ${ }^{85}$

Der Staat bemühte sich, mit Auflagen zu Größe von Grundstück und Wohnfläche den Prozess unter Kontrolle zu behalten: Je nach Region und Örtlichkeit durften die Grundstücke zwischen 300 und 600 Quadratmetern in der Stadt und zwischen 700 und 1200 Quadratmetern außerhalb der Stadtgrenzen nicht überschreiten. Im Jahr 1958 wurde zudem festgelegt, dass die Wohnfläche maximal 60 Quadratmeter betragen durfte. Diese Norm konnte jedoch durch die Größe der Familie bzw. durch Sonderrechte, die an bestimmte Gruppen wie Kriegsveteranen, hohe Militärs oder höhere Fachangestellte vergeben wurden, erhöht werden.

Die Parzellenzuteilung konnte per Antrag beim städtischen Ispolkom oder über den Arbeitgeber-Betrieb erwirkt werden. Eigenheimbau war eine begehrte und häufig auch die einzige Option, die eigene Wohnsituation zu verbessern, und so barg die Frage der Zuteilung reichlich Spekulations- und Konfliktpotential. Die Unübersichtlichkeit der Lage und die Abwesenheit von einheitlichen Wartelisten

83 Andrusz 1984, S. 100.

84 Andrusz 1984, S. 100 u. 290-291.

85 Stronski 2012, S. 316 Fn. 67. 
provozierten Situationen, bei denen Einige „vergessen“ und Andere bevorzugt wurden - sei es durch bloße Zugehörigkeit zu einem wichtigen Betrieb oder durch sonstige Modelle von Klientelismus und informellen Beziehungen, wie den notorischen blat. ${ }^{86}$ Da die Zuteilung staatlichen Wohnraums von den selben Problemen gekennzeichnet war, bemühten sich Politik wie Behörden nach und nach, Ordnung in die Wartelisten zu bringen. In Leningrad wurden die ersten diesbezüglichen Versuche bereits Mitte der 1950er-Jahre unternommen. ${ }^{87}$ Die Ansätze gingen nicht immer in Richtung Zentralisierung. So wurde zum Beispiel in Taschkent 1969 die Listenführung von den Bezirks-Ispolkoms als Aufgabe an die Arbeitgeber weitergereicht, was eigentlich Dezentralisierung bedeutete. ${ }^{88}$

Neben den Problemen mit der Zuteilung wurde der individuelle Wohnungsbau auch durch infrastrukturelle Schwierigkeiten begleitet. In den Fällen, wo der tatsächliche Neubau nicht mit den Plänen oder den Kapazitäten der städtischen Behörden übereinstimmte, wo Interessenskonflikte zwischen der Stadtverwaltung und anderen Akteuren - wie industriellen Betrieben oder umliegenden Kolchosen - bestanden, oder wo man einfach durch allgemeinen Mangel an Finanzen und Arbeitskraft ins Hintertreffen geraten war, wurden die so genannten „Viertel der planmäßigen individuellen Bebauung“ häufig stiefmütterlich behandelt. Oft wurden schlecht geeignete bzw. abgeschiedene Territorien zugewiesen. Für Klagen und Kritik sorgte auch die mangelhafte oder ganz fehlende Bereitstellung von Infrastruktur, ${ }^{89}$ was die Wohnqualität in solchen Vierteln minderte.

Während die gesetzlichen Rahmenbedingungen das individuelle Bauen in den 1950er-Jahren förderten, waren es letztlich die Ressourcen und Kapazitäten vor Ort, die für die Umsetzung ausschlaggebend waren. Auch auf der obersten Regierungsebene waren die Meinungen über die Rolle bzw. die Folgen einer konsequenten Umsetzung sehr unterschiedlich. Auf der einen Seite standen die Befürworter, darunter der Leiter des Bauministeriums Gosstroi, Vladimir Kucherenko, die den Sektor weiter ausbauen wollten und unterschiedliche Vorschläge ausarbeiteten, wie das System effektiver gestaltet werden könne. Das schloss eine bessere Versorgung mit Baumaterialen sowie höhere Kredite bzw. längere Rückzahlungsfristen ein. ${ }^{90}$ Zudem haben einige sowjetische Stadtforscher darauf hingewiesen, dass ein Grundstück mit Garten für Neuankömmlinge vom Land in

86 Smith 2010, S. 94. Zum Phänomen des sowjetischen blat siehe Ledeneva 1998.

87 Smith 2010, S. 94.

88 Biryukov 1971.

89 Andrusz 1984, S. 103

90 Smith 2010, SS. 90, 161-162. 
den Städten eine Überlebensfrage war. ${ }^{91}$ Auf der anderen Seite befanden sich die Kritiker, die von unterschiedlichen Standpunkten her argumentierten. Neben der ideologischen Kritik, die den individuellen Wohnungsbau als Förderung eines kleinbürgerlichen Lebensstils und des Missbrauchs von Parzellen für Anbau und so genannten spekulativen Verkauf von privater agrarischer Produktion verurteilte, wurden auch im engeren Sinne ökonomische Gründe wie die Verschwendung von Bauland und die sehr hohen Kosten für die Bereitstellung von Infrastruktur bei geringer Dichte der Bebauung angeführt. Zum anderen wurden ästhetische Argumente vorgebracht, da der Anblick solcher individuellen Bauten nicht dem Bild einer modernen Stadt entsprochen habe. ${ }^{92}$

Insgesamt wurde der individuelle Hausbau, so Andrusz, vom Staat als „,notwendiges Übel“ betrachtet, das toleriert und teilweise gefördert wurde, solange der Staat nicht in der Lage war, den Bedarf an Wohnraum alleine einzulösen. Außerdem brachte er private Ersparnisse der Bürger in Umlauf - zum Beispiel beim Ankauf von Baumaterialien. Gleichzeitig benutzte der Staat bestimmte Formen der kollektiven Organisation für propagandistische Zwecke: Die lokalen Initiativen, bei denen Kollegen eines Betriebs gemeinsam für sich selbst Wohnungen und Häuser bauten - was als narodnaya stroika „Volksbaustelle“ oder metod gor'kovchan „Gorky-Methode“ bezeichnet wurde - wurden groß propagiert und als ein Akt des „hohen sozialistischen und kollektiven Verantwortungsbewusstseins “ gefeiert. ${ }^{93}$

Der individuelle Bausektor hatte auch eine Schattenseite in der Gestalt von nicht autorisiertem Bauen, das in der UdSSR als samovol'noe stroitel'stvo bezeichnet wurde. Später in dieser Arbeit werde ich am Beispiel von Samarkand noch intensiv auf dieses Phänomen eingehen. Mark Smith erweitert Andrusz' Beobachtung vom „notwendigen Übel“, indem er verstärkt auf die Perspektive der Bürger eingeht und das individuelle Bauen, offizielles wie inoffizielles, sowie andere legale wie illegale Strategien der Wohnraumbeschaffung als Ausdruck von „permitted autonomy“ identifiziert. Ihm zufolge war all dies „systemic to the urban housing economy“, insbesondere in der Periode zwischen 1944 und 1964.

Die Wende bahnte sich bereits 1958 an, als der kooperative Wohnungsbau erneut erlaubt und schrittweise auf die Agenda geholt wurde. ${ }^{94}$ Der industrielle

91 Svetlichnyi in DiMaio 1974, S. 28.

92 Andrusz 1984, S. 104.

93 Eine Initiative, bei der sich Kollegen gegenseitig bei der Errichtung der Häuser halfen, wodurch diese recht schnell gebaut werden konnten. Ausführlicher über das Phänomen der „Gorky-Methode“ und die damit verbundene Kampagne bei Harris 2013, S. 154-171.

94 Smith 2010, S. 108 
Wohnungsbau gewann parallel immer mehr an Stärke, sodass der Staat hier allmählich zum Hauptbauherrn wurde und die Hauptlast der Wohnungsversorgung von den Betrieben und Organisationen übernehmen konnte. Die damit einhergehende Zentralisierung sollte eine bessere Kontrolle sowohl über den Bauprozess und dessen Kosten als auch über die Wohnungsverteilung erlauben. Damit konnte sich der Staat von der Notlösung des individuellen Wohnungsbaus zunehmend emanzipieren. Der 1962 erschienene Erlass „Über den individuellen und kooperativen Wohnungsbau“"95 verkündete den Übergang vom individuellen zum kooperativen Wohnungsbau und verhängte ein Verbot der Parzellenzuteilung in den Republikhauptstädten. Die Parteiorganisationen, Räte und Betriebe sollten die Wende unterstützen, indem sie verpflichtet wurden, die Bevölkerung in Aufklärungsgesprächen für die kooperative Form des Bauens zu gewinnen. ${ }^{96}$ Die Entscheidung über die Parzellenzuteilung in Städten mit über 100.000 Einwohnern sollten die lokalen Ministerräte treffen. In der RSFSR wurde 1963 die Parzellenverteilung in solchen Städten untersagt. ${ }^{97}$ Das individuelle Bauen blieb damit den kleineren Städten und Siedlungen vorbehalten, wo sich der Aufbau einer speziellen Bauindustrie nicht lohnte. Der unionsweite durchschnittliche Anteil des individuellen Sektors am Neubau sinkt in den darauffolgenden Jahren von 24\% oder 14 Mio. $\mathrm{m}^{2}$ im Jahr 1960 bis 8\% (6 Mio. $\left.\mathrm{m}^{2}\right)$ im Jahr 1980. ${ }^{98}$ Das Beispiel der Usbekischen Republik, über den in den nächsten Kapiteln ausführlicher berichtet wird, macht jedoch deutlich, dass der Prozess nicht einheitlich war.

Sowohl Smith als auch Andrusz verbinden die Wende im Wohnungsbau mit der persönlichen Einstellung Khrushchevs. Sie nehmen an, dass er seine Überzeugung durchsetzen wollte, den Übergang von Sozialismus zu Kommunismus voranzubringen, indem der Wohnraum in den Städten den sozialistisch-kommunistischen Idealen angepasst wurde. ${ }^{99}$ So bezeichnet Smith den Abriss individueller Häuser im Zuge des Wachsens von mikroraions als „[...] an ideological attack on personal property as a legal category and the individual urban house as the symbol of a backward-looking way of life." ${ }^{100}$ Den Wechsel in der Politik gegenüber dem Eigenheim allein in ideologischen Beweggründen Khrushchevs zu

95 Erlass des ZK der KPdSU vom 01.06.1962.

96 Diese Wohnungsbauform kann man als Aktivität von staatlich initiierten Baugenossenschaften verstehen, bei denen die bei der Bevölkerung vorhandenen Mittel und Ersparnisse nicht mehr für Eigenheime, sondern für den Bau von mehrgeschossigen Wohnhäusern benutzt werden sollten.

97 Erlass des Ministerrates der RSFSR vom 03.09.1963.

98 Andrusz 1984, S. 21, Tabelle 1.4.

99 Andrusz 1984, S. 100-101.

100Smith 2010, S. 162. 
sehen, greift jedoch zu kurz. Dies kann nur ein Teilaspekt der Entscheidungen des Politbüros gewesen sein. Eine Mitschrift der Sitzung des Präsidiums vom 17.06.1961, bei der die Wohnungsfrage diskutiert wurde, zeigt ein etwas anderes Bild. ${ }^{101}$ Aus ihr wird deutlich, dass das Hauptinteresse Khrushchevs in der Maximierung und Optimierung des Wohnungsbaus lag. Seine Kritik am individuellen Wohnungsbau richtet sich vor allem gegen die hohen Kosten für die Bereitstellung von Infrastruktur; die Idee des individuellen Hausbaus und die Tatsache, dass Menschen sich auf eigene Kosten solchen Wohnraum errichteten, fand bei ihm angesichts des Wohnungsmangels sogar Verständnis. Khrushchev zufolge würde eine staatliche finanzielle Förderung jedoch dem Geist des industriellen Wohnungsbauprogramms widersprechen; Ideologie im Sinne der kommunistischen Kollektivierung spielte, zumindest nach Ausweis dieses Dokuments, keine Rolle. Es waren aber der Massenwohnungsbau und die groß angelegte und imaginierte Technologie, die in den Rang einer Ideologie gehoben wurden. ${ }^{102}$

Insgesamt setzten sich die Gründe für die Abkehr von der intensiven Förderung des individuellen Hausbaus unter Khrushchev aus unterschiedlichen Erwägungen zusammen. Die Frage der Kosten, Praktikabilität und Infrastruktur sowie Einwände von Seiten der Stadtplaner dürften gleichermaßen eine Rolle bei den entsprechenden Entscheidungen gespielt haben. Die letztlich gefundenen Lösungen dieser Frage konnten sich von Ort zu Ort erheblich unterscheiden, wie ich am Beispiel von Samarkand zeigen werde.

101 Fursenko (Hg.) 2015, S. 536.

102 Josephson 1995, S. 520. 